\title{
Opinión
}

\section{Percepción de la obesidad en adultos costarricenses}

Gioconda Padilla- Vargas, Marlene Roselló- Araya, Sonia Guzmán Padilla, Ana Gladis Aráuz Hernández.

\section{Resumen}

La obesidad es un problema de salud que afecta a individuos en cualquier edad y se ha identificado como un factor de riesgo común a muchas enfermedades crónicas. La última encuesta de nutrición realizada en Costa Rica señala que en el grupo de mujeres de 20 a 44 años de edad la prevalencia de obesidad fue del $45,9 \%$, un $11,3 \%$ más que en 1982 ; y en el grupo de 45 a 59 años, la prevalencia fue del $75 \%{ }^{1}$. En la encuesta de Damas en Desamparados, el sobrepeso u obesidad en mujeres de 19 a 44 años fue del 56,4\% y el 58,8\%, en hombres de 19 a 59 años; la encuesta CARMEN sobre factores de riesgo encontró un 59,4\% de la población adulta entre 20 y 64 años, con problemas de sobrepeso u obesidad. (Ministerio de Salud. Encuesta basal de factores de riesgo para enfermedades no transmisibles. Cartago 2001. San José, Costa Rica: Ministerio de Salud, 2003. Serie de documentos técnicos \#4 documento no convencional.

Descriptores: Percepción del peso, obesidad, Costa Rica.

Key words: Overweight perception, obenty, Costa Rica.

En el origen de esta patología se han identificado determinantes biológicos, psicológicos y ambientales y algunos de ellos pueden constituirse en barreras para su control y prevención. Algunas barreras socioculturales para controlar la obesidad son la negación del exceso de peso por parte de los individuos, el desconocimiento del riesgo que este presenta para la salud, así como la influencia social en relación con una figura socialmente aceptable (idea social).

Históricamente, el exceso de peso se ha asociado con bienestar económico y belleza; asimismo, se definen figuras modelo como prototipo al que deben aspirar los individuos según el sexo y la etapa de desarrollo. Se promueve y refuerza la imagen obesa en el área infantil, asociándola con salud y belleza. Por el contrario, en la mujer adolescente y adulta joven se explota la figura delgada y en la edad madura se evoca y acepta la imagen corporal con sobrepeso. En algunas culturas latinoamericanas, la ganancia de peso en la mujer después del matrimonio es un reflejo positivo de bienestar. Esta ambivalencia genera conflicto en la percepción de la imagen corporal; hay confusión desde lo simbólico y lo imaginario.

La percepción tiene que ver con "una valoración que el individuo hace a partir de la información sensorial que ha adquirido, al ver y escuchar los patrones significativos forjados en la infancia, desde los cultural y social" ' ${ }^{2}$. Se relaciona con sentimientos y apreciaciones del mundo interno y externo del individuo. La percepción que una persona tiene sobre un aspecto en

Instituto Costarricense de Investigación y Enseñanza en Nutrición (INCIENSA)

Correspondencia: INCIENSA Apdo 4-2250 TRES RIOS, e-mail gpadilla@inciensa.sa.cr

ISSN 0001-6002/2006/48/3/129-130 Acta Médica Costarricense, $\odot 2006$ Colegio de Médicos y Cirujanos particular, está influenciada por la percepción social, la percepción simbólica, la percepción imaginaria y la percepción de la salud. Desde esta perspectiva, nos encontramos ante un gran reto en lo referente al tratamiento de la obesidad, ya que además de los aspectos meramente fisiológicos, el psicológico influye en gran medida en éxito o fracaso.

En un análisis exploratorio realizado en el INCIENSA en 1999-2000, se valoró la percepción de la imagen corporal a través de una serie de figuras que se han utilizado en contextos semejantes en personas con sobrepeso, con bulimia y con peso normal ${ }^{3}$. Se encontró que las mujeres con sobrepeso tienen alterada la percepción de su imagen real. Según el índice de masa 
corporal (IMC: peso en kilogramos dividido entre la estatura en metros cuadrados), las mujeres se perciben con un peso corporal inferior al real (IMC percibido entre 25$28 \mathrm{~kg} / \mathrm{m}$ vs IMC real entre $28-35 \mathrm{~kg} / \mathrm{m}$ ) (Ministerio de Salud, CCSS, INCIENSA, IAFA. Encuesta multinacional de diabetes mellitus, hipertensión arterial y factores de riesgo en el Área Metropolitana, 2004 (datos preliminares, inéditos), y desean adquirir una figura con IMC entre $19 \mathrm{y}$ 22. Se observó como lo social se impone al desearse adquirir una imagen ideal (percepción imaginaria), a veces casi inalcanzable (figura muy delgada). Por otra parte, al visualizarse una persona con un peso menor al real, podría tener dificultad de ver las consecuencias del exceso de peso, ya que los problemas de salud por obesidad "se visualizan en otros, y no en la misma persona". Es como una manera de negar (desde lo simbólico), la existencia de un problema.

En esta misma exploración, se solicitó a los participantes que le dieran nombre a cada una de las figuras utilizadas en la escala. Llama la atención el no uso de la palabra "obeso", principalmente en mujeres; por el contrario, son frecuentes las palabras "rellenita(o)", "gorda(o)", "gordita", "hermosa(o)" o "muy gorda(o)". Pareciera que existe adversidad ante el uso de la palabra "obeso", situación que dificulta trabajar con esta enfermedad, pues desde lo simbólico se rechaza, se niega su existencia.

Datos similares se obtuvieron más recientemente, cuando el INCIENSA, en coordinación con el Instituto de Estudios Sociales en Población (IDESPO) ${ }^{5}$, incorporó un módulo de cinco preguntas sobre el control del peso corporal en la Primera Encuesta Telefónica de Salud del IDESPO5, con representatividad nacional. Se consultó a 600 costarricenses acerca de cómo perciben su peso, y se determinó que solo el $42 \%$ se percibe con "peso alto o muy alto". Se calculó el IMC a partir de peso y talla autoreportados y se encontró que más de la cuarta parte $(27,7 \%)$ de los hombres entrevistados que se perciben con "peso normal", presentan sobrepeso u obesidad; situación similar ocurre con el $22,5 \%$ de las mujeres, incluso un $14,3 \%$ de las que se perciben con peso bajo o muy bajo presentan un IMC $>$ a 25 .

Pareciera que un porcentaje importante, tanto de hombres como de mujeres, no reconocen el exceso de peso como un problema de salud real, tal es así que en la última encuesta sobre Diabetes y factores de riesgo realizada por el Ministerio de Salud en 2004, se encontró que el 60\% de los entrevistados presenta sobrepeso u obesidad (Ministerio de Salud, CCSS, INCIENSA, IAFA. Encuesta multinacional de diabetes mellitus, hipertensión arterial y factores de riesgo en el Área Metropolitana, 2004 (datos preliminares, inéditos). A partir de esta información se reconoce un subregistro del problema del exceso de peso, desde cómo los costarricenses perciben su imagen corporal y su propio peso, y sobre cómo influye esta percepción en las decisiones cotidianas para enfrentar la obesidad.

¿Cómo atacar un problema cuando este no se reconoce? El reto es grande para las instituciones de salud costarri- censes, más aún cuando los mismos proveedores de salud tienen la enfermedad e igualmente no siempre la reconocen, lo que refiere un problema complejo que toca a la población en general.

Tradicionalmente, el personal de salud percibe este problema de manera distinta, más biologista, se podría decir; se da énfasis a la relación de salud física y enfermedad, lo que confunde a las personas que la padecen, ante la no coincidencia entre la percepción de la obesidad desde la salud y desde lo simbólico, imaginario y social.

Ante la demanda social, donde contradictoriamente se exige una figura delgada en un ambiente que facilita el sobrepeso y la obesidad, la persona con este problema experimenta altos niveles de frustración. Las ilusiones de percibir una figura muy delgada, que en la realidad no existe o que es muy difícil de adquirir, hace que se pida a la persona afectada algo visto como un posible: un peso ideal que en ocasiones dista mucho del peso real que una persona con serios problemas de sobrepeso podría alcanzar. El resultado de esta confusión siempre es "un daño físico, psíquico, social, económico o, una combinación de todos ellos" 5 .

Considerar tales elementos en la reflexión sobre el abordaje de esta enfermedad, es un reto para ampliar el enfoque tradicional que se utiliza en el tratamiento de la obesidad, de manera que se geste un enfoque más integral y real, que permita acercarse a la obesidad, no solo desde lo físico o cuantitativo, sino también desde las vivencias de las personas y los significados que se le adjudican, que de una $\mathrm{u}$ otra forma pueden obstaculizar o favorecer su control.

\section{Abstract}

Obesity is a health problem that effects individuals of any given age group, and it has been identified as risk factor, common to many chronic diseases. The lastest nutrition poll carried in Costa Rica shows that the prevalence of obesity in women 20 to 44 years old was $45.9 \%$, $11.3 \%$ higher than in 1982 and in the group of 45 to 59 years, the prevalence was 75\%1. According to the findings of the Damas/Desamparados poll overweight in women 19 to 44 years was $56.4 \%$, and in men 19 to 59 years was $58.8 \%$, the Carmen poll regarding risk factors found that $59.4 \%$ of the adult population (20-64 years) had overweight or obesity.

\section{Referencias}

Ministerio de Salud. Encuesta nacional de nutrición: Antropometría. Costa Rica: Ministerio de Salud, 1996. Fascículo 1.

2. Morris Ch. Psicología: Un nuevo enfoque. $7^{\circ}$ ed. México: PrecticeHall Hispanoamericana, 1992.

3. Welch Y. Prevalencia de trastornos de la conducta alimentaria en secundarias y preparatorias laicas y religiosas de la ciudad de México. Tesis UIA: México, DF, 1998.

4. Instituto de Estudios Sociales en Población (IDESPO). Percepciones de la ciudadanía costarricense sobre estilos de vida saludable y los servicios de salud. Pulso Nacional: 35. Heredia, Costa Rica: Universidad Nacional de Costa Rica, 2004.

Alemany M. Obesidad: Una epidemia con componentes estéticos. Obesidad y nutrición. 1998; 1: 184-187. 